\title{
Palm print Classification based on inter-Distal Region Texture features
}

\author{
Dhananjay D.M \\ Research Scholar \\ JNTU \\ Hyderabad,India
}

\author{
C. V. Guru Rao, PhD. \\ Professor \& Head \\ Department of CSE \\ S.R.Engineering College \\ Warangal, India
}

\author{
I. V. Muralikrishna, PhD. \\ Former Director, $\mathrm{R} \& \mathrm{D}$ Cell \\ JNTU \\ Hyderabad,India
}

\begin{abstract}
Palm print is a widely accepted biometric trait for authentication due to clarity in discriminating the features of palm such as large distance among non-class samples as well as minimum distance between intra-class samples. Area beneath the finger and enclosed by heart line is called as triradiated region or inter-distal region. This specific area of palm contains features which are unique and universally discriminating. In this paper we present a simple method to extract ridges in tri-radiated section also called as inter-distal region of palm. Different orientation of ridges extracted from inter-distal region appears as a fine texture. We use these fine textures for validating samples of Palm print. Reduction in size of the image, it's optimal storage, retrieval, computational efficiency without compromising the fine features of the palm sample and use of simple discriminating features to validate a given palm sample has motivated this paper. The results confirm the proposed methodology in this paper is most efficient one.
\end{abstract}

\section{General Terms}

Pattern Recognition, Digital forensic.

\section{Keywords}

Inter-distal region, Tri-radiated section, low pass filter, Gabor filter, texture feature, Manhattan distance.

\section{INTRODUCTION}

Palm print based authentication is being used commercially for establishing identity for secured access of resources [1]. When compared to finger print, palm print has not gained wider acceptance due to the sample size. A solution widely followed to reduce the area of palm sample is to select a square region by using a universally accepted reference point. One such reference point being used is parallel line drawn below the base of fingers to extract region of interest (ROI) [2] as shown in Figure 1. A submitted palm sample can be interpreted as collection of different regions or section demarcated based on the principle lines or major lines of the palm sample. Three principle lines that are prominent in every palm print are Heart line, Head Line and Life line [3]. The heart line normally originates from area below the small finger and terminates below the index finger and is also known as distal crease. Major regions which make $75 \%$ of the palm print sample area are Thenar region, Hypothenar region and Tri-radiated region. Terms inter distal region and triradiated section or region are used interchangeably in this paper. They are also referred as Region-I, Region-II and Region III respectively. Region-I or Tri-radiated section is area beneath the base of the finger enclosed by Heart line. The area enclosed by life line is known as Thenar region. Area opposite to Thenar region is known as Hypothenar region. The area enclosed by this line below the base of finger is called as Tri-radiated section. Palm sample shown in Figure 2 depicts different region or sections of palm print along with principle lines. A close observation of tri-radiated section reveals that this region has good number of features to differentiate between individual palm print samples. Some of the features that can be captured in tri-radiated section are ridges, whorl, arch, minutia lines etc. If we use tri-radiated section as ROI to compare two samples, size of sample will be limited at the same time we have good number of features to validate a given sample. In this paper we use tri-radiated section to represent palm print sample and propose a computationally effective method for validation of palm print sample.

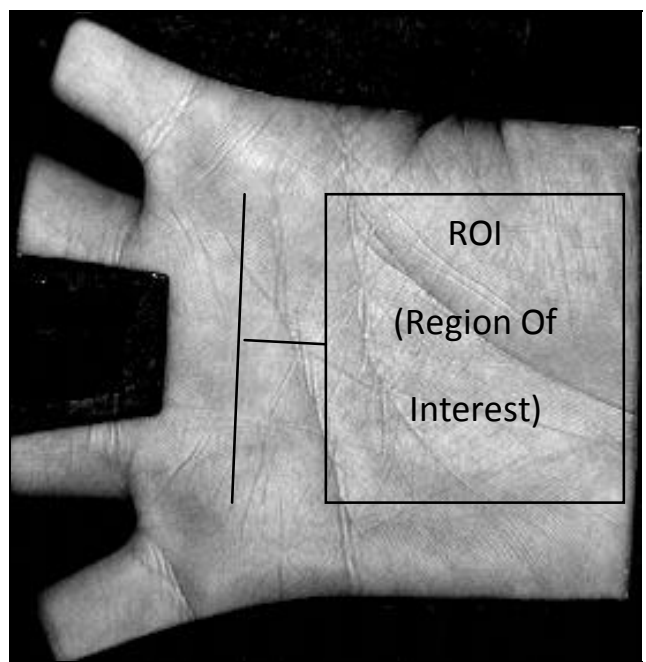

Figure1. Region of Interest (ROI) in sample Palmprint (Courtesy: PolyUDatabase)

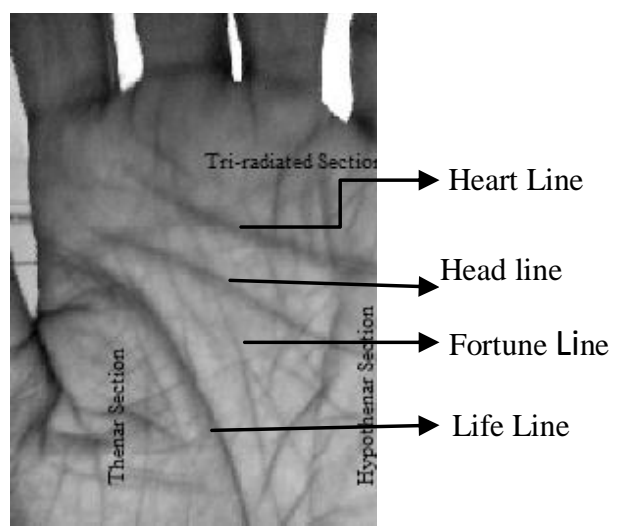

Figure 2. Palm print Regions and lines In this paper section 2 describes different features of triradiated region. Pre-processing of sample is presented in section 3. Section 4 explains the use of Gabor filter in six 
different orientations for generating sub samples. Simple texture based feature extraction method is illustrated in section 5. Section 6 analyzes the results obtained on the proposed method. The research work presented in this paper is concluded in Section 7 followed by the references.

\section{FEATURES OF TRI-RADIATE REGION}

Tri-radiated section occupies around $20 \%$ of the total area of palm print. Area beneath the index finger up to little finger, and below the heart line is designated as tri-radiated section or region. The theory of palmistry regards this area as the one containing maximum features than compared to any other area. A close observation of this area reveals that many features which can be studied with finger print are also present in this area. While finger print normally works with thumb sample as finger print, Ti-radiated section has multiple such areas adjoining to each other. This feature can be useful to increase the reliability of the palm print authentication process. Ridge features, minutiae features, whorl formation of ridge, arch formation, frequency of ridges in given area, orientation of ridges in different area, ending point of ridges, formation of island due to ridges and crossing of ridges are some of the useful features that are useful parameters to be used to discriminate one region from other region[4]. Another advantage with this area is the listed features are rotation and scale independent. Further the methods which are well established in finger print authentication process can be directly used here. As mentioned in earlier discussion the size of sample will also be optimal hence enhancing the authentication computational process. A sample area of triradiated section / inter distal region captured using conventional method is shown in fig (3).

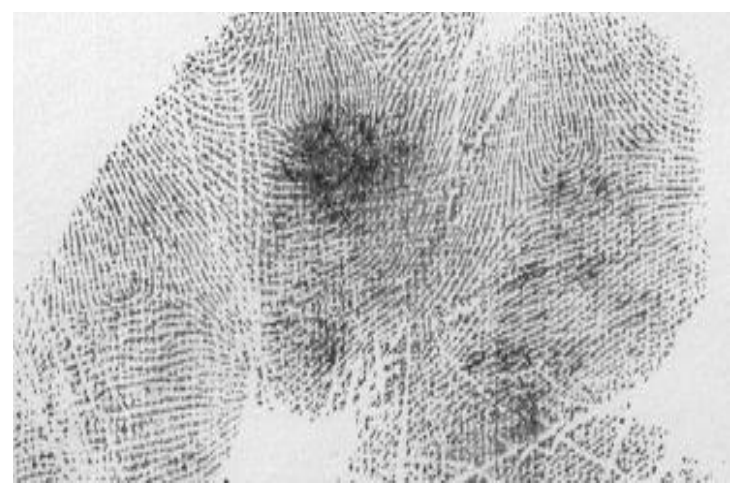

Figure3. Sample Inter-distal/Tri-radiated Section

\section{PRE-PROCESSING OF TRI-ADIATED REGION}

For pre-processing of sample palm print we use a global threshold value to binarize the image sample using eqn (1). This process removes the background pixel [5]. We apply low pass filter [6] to remove high frequency components using eqn(2).

$$
\begin{aligned}
& I M= \begin{cases}1 & \text { if } \operatorname{im}(i, j)>T \\
0\end{cases} \\
& I M=i m(i, j) \otimes \text { LowPass Filter }
\end{aligned}
$$

Result of this operation is shown in fig $(4 a, 4 b)$.

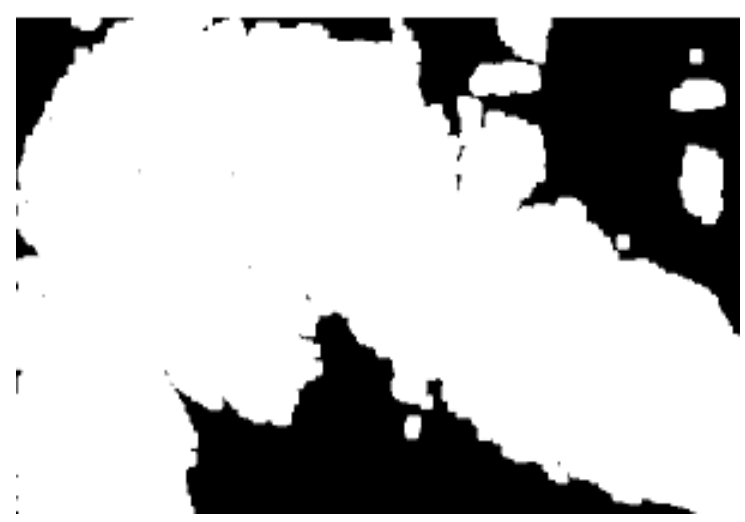

Figure4a. Input sample Binarized

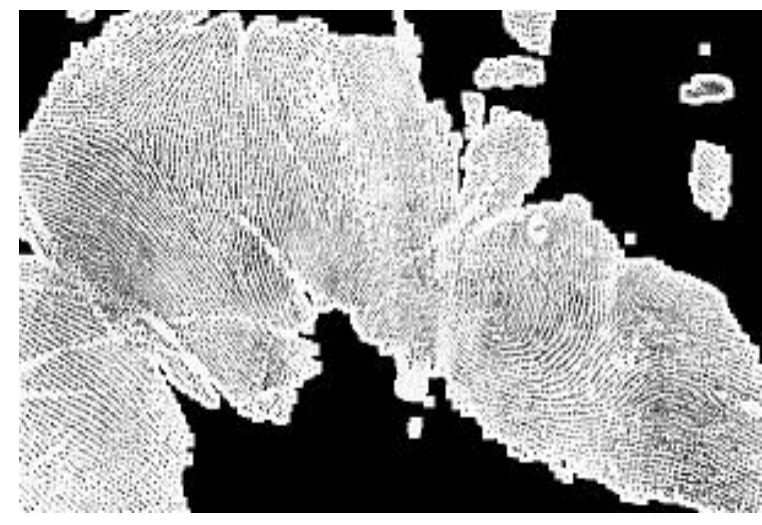

Figure4b. Filtered Sample

\section{SUB SAMPLE GENERATION USING GABOR FILTER}

Pre processed palm print image samples are applied to the Gabor filter using six orientations to generate sub samples in six directions. Response of ridges in specific direction is consolidated and is used for feature extraction using texture calculations. Gabor filter, Gabor filter bank and Gabor wavelet are being used in feature extraction in image processing due to their fine scale response controlled by different orientation and scale [9]. A general Gabor expression for spatial domain is as given eqn (3).

$$
G(x, y, u, \theta)=1 / 2 \pi \sigma e^{\left(x^{2}+y^{2}\right) / 2 \sigma} e^{(u x i \sin \theta+u y i \cos \theta) / 2 x y}
$$

Where $\mathrm{i}=\sqrt{-1}, \mathrm{u}$ is the central frequency of the sinusoidal wave and in spatial domain controls the pixel resolution, $\theta$ controls the orientation of the function and $\sigma$ is the standard deviation of the Gaussian envelope. We use $\theta$ in six orientations to capture the response of the filter. The six directions used are $(0,30,60,90,120$, and 150) the other directional responses are mirror image of the directional set used. Following Figure 5 shows the process used for obtaining the filtered image in six directions further the images are applied threshold to obtain the required response. 


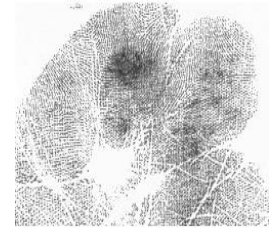

a. Input Sample

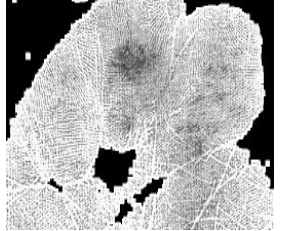

b. Pre-processed sample

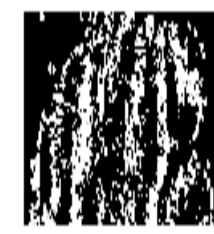

c. 0 Degree

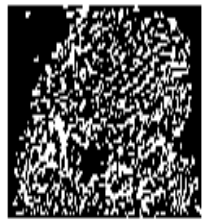

f. 90 Degree
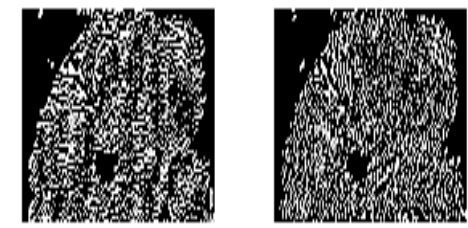

d. 30 degree

e. 60 degree
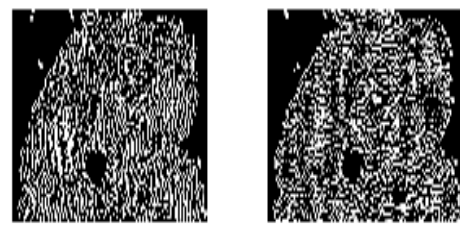

g. 120 Degree

h.150 degree

Figure 5. a) Input Sample b) Pre-processed sample c) to $h)$ Gabor filter response

\section{TEXTURE BASED FEATURE}

\section{EXTRACTION AND PALMPRINT CLASSIFICATION}

\subsection{Texture feature extraction.}

Response obtained from Gabor filter in six directions depicts a fine distribution of ridge orientation with respect to direction $\Theta$. This fine distribution of ridge response appears as a spatial domain texture distribution. Texture can be defined as a regular repetition of an element or pattern in a given image[10]. Texture features are studied under statistical domain and syntactic domain [7] [8]. In our paper we have used five texture features Energy, Entropy, Contrast, Homogeneity and Correlation defined by eqn $(4,5,6,7,8)$ respectively.

$$
\begin{aligned}
& \text { Energy }=\sum_{i} \sum_{j} p d^{2}(i, j) \\
& \text { Entropy }=\sum_{i} \sum_{j} p d(i, j) \log p d(i, j) \ldots(5) \\
& \text { Contrast }=\sum_{i} \sum_{j}(i-j) p d(i, j) \\
& \text { Homogeneity }=\sum_{i} \sum_{j} p d(i, j) /(1+|i-j|)
\end{aligned}
$$

$$
\text { Correlation }=\sum_{i} \sum_{j}\left(i-\mu_{x}\right)\left(j-\mu_{y}\right) p d(i, j)
$$

For each direction of $\Theta$ degree Gabor filter response is obtained. As we use six different values for $\Theta$ we get six responses for each sample and calculate five texture features[11] as mentioned in eqn $(4,5,6,7,8)$ given above. This results in generating rich texture feature vector. The following table (1) depicts the texture feature calculated for each direction for one of the sample palmprint from data base.

Table1. Feature Vector calculated for sample-1

\begin{tabular}{|l|r|r|r|r|r|r|}
\hline $\begin{array}{l}\text { Direction } \\
\text { Feature }\end{array}$ & 0 & 30 & 60 & 90 & 120 & 150 \\
\hline Energy & 326380 & 152200. & 156980. & 360150. & 214770. & 160160. \\
\hline Entropy & 99733.0 & 36399.0 & 38611.0 & 107840. & 62456.0 & 39342.0 \\
\hline $\begin{array}{l}\text { Homogeneit } \\
\mathbf{y}\end{array}$ & 2009.00 & 1446.00 & 1361.00 & 2081.00 & 1516.00 & 1422.00 \\
\hline Contrast & 0.06 & 0.04 & 0.04 & 0.05 & 0.05 & 0.04 \\
\hline Correlation & 2009.00 & 1446.00 & 1361.00 & 2081.00 & 1516.00 & 1422.00 \\
\hline
\end{tabular}

\subsection{Palm Print Classification Based On Texture Features.}

Feature vectors obtained for each sample are used for finding the class of palmprint. We have used Manhattan distance [12] given in eqn(9) classifier for assigning proper class to palmprint. If $\mathrm{P} 1$ is one point at coordinate $(\mathrm{x} 1, \mathrm{y} 1)$ and $\mathrm{P} 2$ is another point at $(\mathrm{x} 2, \mathrm{y} 2)$ then the distance between two points $\mathrm{P} 1, \mathrm{P} 2$ is given by

$$
\text { Manhattn Dist }=|x 1-x 2|+|y 1-y 2|
$$

For each sample and each directional response distance Di for $\mathrm{i}=1: 6$ is calculated and the final classification will be based on finding out the different samples to which the sample is nearest. The maximum number of time a sample $\mathrm{Si}$ is closer to submitted palmprint from database stored, that sample is obtained as the final class of the sample.

\section{RESULTS AND DISCUSSION}

\subsection{Results}

We have used samples collected using conventional inking method. In this method ink pad ink is spread on Inter Distal region and the palm is pressed against a blank good quality paper. Three instances of same sample at different times are used. The samples are scanned at 300 dpi. Format for the image used is BMP. We have calculated for each sample based on texture feature which sample is nearest. Results of this calculation are presented in table (2). If a Sample $S_{i} \in$ MaxOccurance $\left\{\right.$ DBSample $\left.{ }_{i}\right\}$ then class of sample is DBSample ${ }_{i}$, where DBSample sample from database and $S_{i}$ is input test sample. Accuracy of our results obtained by our proposed method is $99.33 \%$. The results obtained for few samples are presented in fig (6). 
Table 2. Samples classified as per texture features

\begin{tabular}{|c|c|c|c|c|c|}
\hline Sample & Entropy & Energy & Contrast & $\begin{array}{l}\text { Homo- } \\
\text { geneity }\end{array}$ & $\begin{array}{l}\text { Cor- } \\
\text { relation }\end{array}$ \\
\hline 1 & 9 & 9 & 12 & 9 & 8 \\
\hline 2 & 4 & 4 & 3 & 4 & 9 \\
\hline 3 & 5 & 5 & 2 & 5 & 4 \\
\hline 4 & 2 & 2 & 5 & 2 & 3 \\
\hline 5 & 3 & 3 & 4 & 3 & 7 \\
\hline 6 & 7 & 7 & 8 & 7 & 8 \\
\hline 7 & 6 & 6 & 9 & 6 & 5 \\
\hline 8 & 6 & 6 & 6 & 6 & 6 \\
\hline 9 & 1 & 1 & 1 & 1 & 12 \\
\hline 10 & 11 & 11 & 11 & 11 & 11 \\
\hline 11 & 10 & 7 & 5 & 10 & 10 \\
\hline 12 & 1 & 1 & 1 & 1 & 9 \\
\hline 13 & 15 & 14 & 14 & 12 & 14 \\
\hline 14 & 13 & 13 & 13 & 15 & 13 \\
\hline 15 & 14 & 12 & 12 & 11 & 12 \\
\hline
\end{tabular}

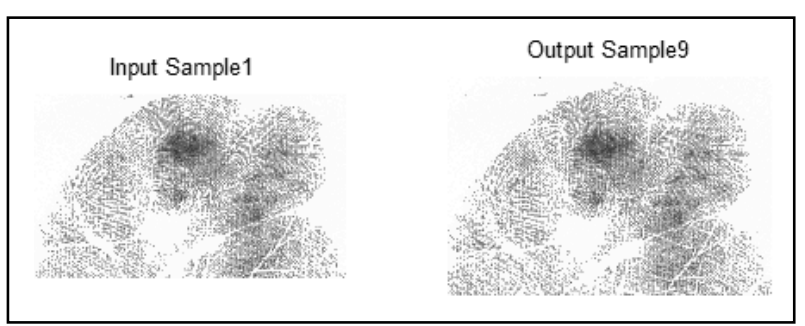

(a)

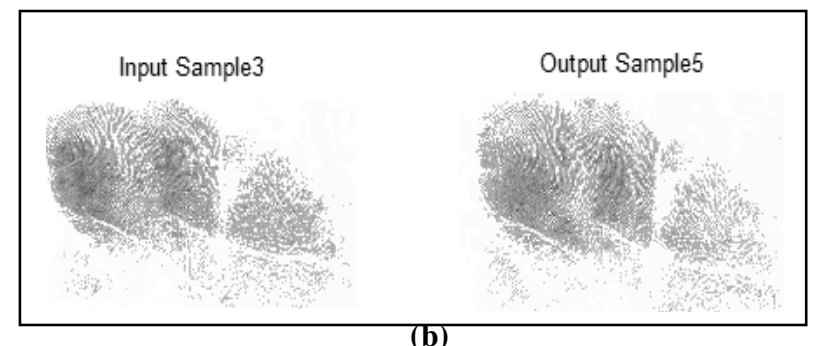

b)

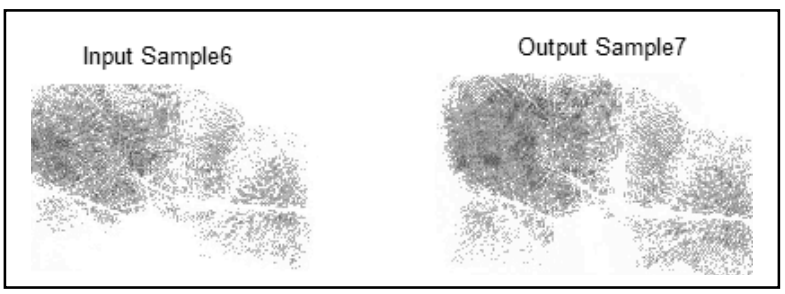

(c)

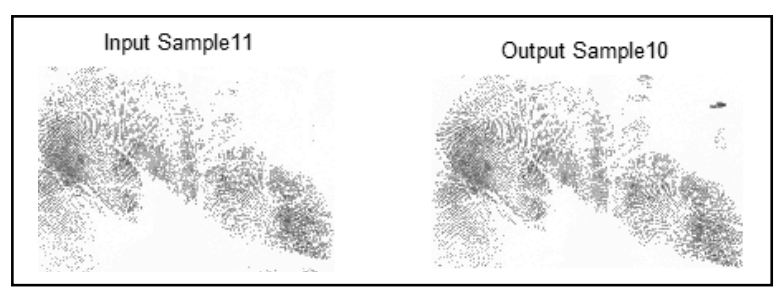

(d)

Figure6. Test results for few samples from database

\subsection{Discussion}

Biometric systems reliability can be assessed by False Rejection Rate which is major of response of a system for valid input sample and number of attempts made by person, This is represented in following graph fig(7). As we change the threshold values to measure FRR, it can be seen that when threshold is increased by $50 \%$ the acceptance rate is 80.33 and the acceptance rate is nearly $60 \%$ when threshold is changed by nearly $200 \%$. This result proves the reliability of system of our proposed method.

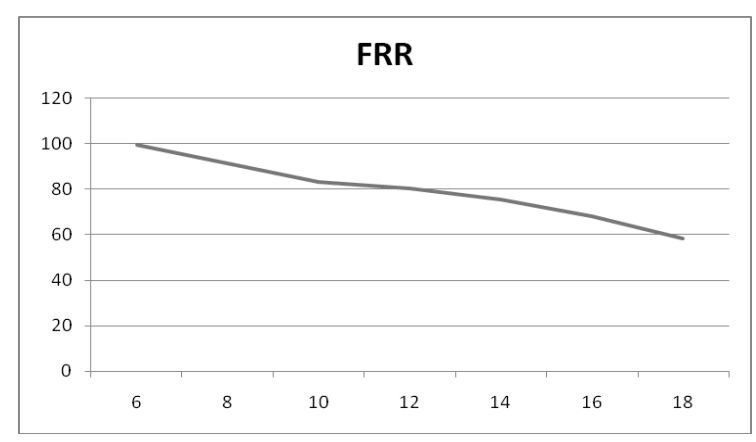

\section{CONCLUSION}

Enforcement of security and authentication is inevitable these days which invariably employ the more effective biometric systems with utmost computational efficiency as well as precision and accuracy. Our proposed palm print based method reduces the computational burden. Further the fine formation of ridge structure has best discrimination features. The system ensures to capture good quality input samples. Texture features are good measure for classification but do not imply a proper ridge formation. The palm print based biometric system is tested with many number of samples and found more efficient than many existing other biometric systems. Therefore it is highly recommended for personal authentication.

\section{REFERENCES}

[1] A.K. Jain, R. Bolle, and S. Pankanti "Biometrics: Personal Identification in Networked Society", Kluwer Academic, 1999.

[2] D. Zhang, A.W. Kong, J. You and M. Wong "Online Palmprint Identification," IEEE Trans. PAMI, vol. 25, No. 9, Sept. 2003 PP. 1041-1050.

[3] H. V. Alexander "Classifying Palm Prints”, Charles C. Thomas Publication, Illinois, 1973162 Pages. 
[4] N. Duta, A.K. Jain, and K.V. Mardia, "Matching of Palmprint", Pattern Recognition Letters, vol. 23, no. 4, 2001 PP 477-485.

[5] Gonzalez, Woods "Digital Image Processing" 3rd Edition Eastern Economy Publication

[6] A.K Jain, Feng, J.J "Latent Palmprint Matching", PAMI Vol. 31, No. 6, June 2009, PP. 1032-1047.

[7] Rafal Kozik and Michal Chora "Combined Shape and Texture Information for Palmprint Biometrics" Journal of Information Assurance and Security vol.5 2010 PP 6066.

[8] Ghandehari, Azadeh, Safabakhsh,"Palmprint verification Using Circular GaborFilter”, ICB09 PP 675-684.
[9] Hans G. Feichtinger, Thomas Strohmer,"Gabor Analysis and Algorithms", Birkhäuser, 1998; ISBN 0817639594.

[10] C. H. Chen, L. F. Pau,P. S. P. Wang "The Handbook of Pattern Recognition and Computer Vision", 2nd Edition pp. 207-248, World Scientific Publishing Co., 1998.

[11] Manesh Kokare, P. K. Biswas, and B. N. Chatterji "Rotation-Invariant Texture Image Retrieval Using Rotated Complex Wavelet Filters", IEEE Transactions On Systems, Man and Cybernetics-Part B: Cybernetics, Vol. 36, No. 6, Dec. 2006, PP 1273-1282.

[12] Richard O. Duda, Peter E. Hart "Pattern Classification", 2nd Edition, Wiley Publication. 